\title{
Aspectos influyentes en la percepción del Aula Invertida de un curso de mercadeo
}

\author{
Influential aspects in the perception of the Flipped Classroom of a marketing course \\ Francisco Javier Beirute Miranda ${ }^{1}$
}

Fecha de recepción: 10-12-2019

Fecha de aceptación: 24-08-2020

\begin{abstract}
Resumen
Para fomentar la innovación didáctica, se decidió implementar, por primera vez, el Aula Invertida en dos cursos de Mercadeo de la Universidad Hispanoamericana de Costa Rica. El objetivo fue analizar la percepción de los y las estudiantes de los cursos de Seminario de Mercadeo 3 con respecto a la estrategia. Se profundizó en cuatro aspectos principales: el rol del profesor, la calidad de los conocimientos, la libertad de expresión durante la clase y el nivel de disfrute del estudiantado. También se analizaron las percepciones acerca de las evaluaciones realizadas en clases. El estudio fue cuantitativo y no experimental. Se utilizó como instrumento de recolección de datos el cuestionario. La valoración del Aula Invertida fue positiva: se consideró que incrementó los aprendizajes, ayudó a memorizar y comprender conceptos teóricos y aumentó el disfrute de las clases.
\end{abstract}

Palabras claves: Aula Invertida, aprendizaje, docencia universitaria, mercadeo, vídeos.

\begin{abstract}
To increase the innovation during the teaching process, Flipped Classroom strategy was used, for the first time, in two Marketing courses at the Hispanoamerican University of Costa Rica. The main goal of the study was to analyze the students perceptions about this strategy in the third Marketing Seminar. The research considered four main aspects: the teacher's role, the quality of the new knowledge, the freedom of expression during class, and the level of students' enjoyment. The perceptions linked to the evaluation process were also taken into consideration for the analysis. This study was quantitative and non-experimental. A questionnaire was used as the main instrument for gathering data. The overall perception of the strategy was positive: it was considered that it increased the learning process, aided concept memorization and understanding, and increased the enjoyment during class.
\end{abstract}

Keywords: Flipped Classroom, learning, university teaching, marketing, videos.

\footnotetext{
1 Mercadólogo, docente e investigador. Universidad Hispanoamericana, Costa Rica. Correo electrónico: j-beirute@uh.ac.cr
} 


\section{Introducción}

Una posibilidad para la construcción de nuevos escenarios de aprendizaje es el Aula Invertida, conocida también como Aula Inversa o Flipped Classroom (en inglés). Esta constituye una alternativa que ha comenzado a estudiarse en distintos contextos universitarios (Albalawi, 2018; Jakobsen \& Knetemann, 2017; Melendo \& Presol, 2018; Motameni, 2018; Wen-Ling \& Chun-Yen, 2017).

Pese a su popularidad internacional, el Aula Invertida ha sido poco estudiada en el contexto costarricense; en particular en el ámbito universitario privado costarricense. En este estudio se implementó la modalidad Aula Invertida en dos cursos de Seminario de Mercadeo 3 de la Universidad Hispanoamericana de Costa Rica, en su sede en la ciudad de Heredia.

El Aula Invertida no había sido utilizada en la Facultad de Ciencias Económicas de la Universidad Hispanoamericana. Se consideró el estudio viable, pues había acceso a la población y se contaba con un cuatrimestre completo para implementar la estrategia didáctica.

Diversos estudios científicos respaldan la credibilidad del Aula Invertida. Entre ellos se encuentra el realizado por Wen-Ling y Chun-Yen (2017), en su investigación Students' perception of a Flipped Classrrom approach to facilitating online project-based learning in marketing research courses. Los autores analizaron la percepción de los estudiantes de Investigación de Mercados en torno al uso del Aula Invertida para facilitar el aprendizaje. Se comenta que el Aula Invertida es un modelo docente que facilita a los estudiantes la adquisición de conocimientos, habilidades y competencias profesionales; además que, desde el punto de vista del estudiante, puede mejorar la efectividad de su aprendizaje. Estos autores consideran que dicha modalidad es una herramienta útil con el fin de lograr un exitoso proyecto docente, tanto desde el punto de vista del docente como por parte del estudiante. En ese estudio, los autores destacan un comentario de un estudiante que percibió la clase como interesante y atractiva, pese a que en un inicio había imaginado que sería aburrida.
En el estudio Una propuesta de Aula Invertida en la asignatura de señales y sistemas de la Universidad Nacional de Colombia, Reyes-Parra, Cañón-Ayala y OlarteDussan (2018) señalan que «los resultados muestran que la percepción de los estudiantes acerca de las actividades desarrolladas utilizando la metodología de Aula Invertida es positiva» (p. 85). Estos autores corroboran la utilidad del Aula Invertida como herramienta docente pues, desde el punto de vista del estudiante, las actividades que se desarrollaron durante el análisis fueron enriquecedoras.

Los autores tomaron en cuenta la investigación ¿Cómo perciben los estudiantes el clima de aprendizaje en el Aula Invertida en cirugía?: Lecciones aprendidas y recomendaciones para su implementación, cuyo objetivo era presentar la medición longitudinal del clima de aprendizaje en el Aula Invertida en cirugía y ofrecer algunas recomendaciones para su implementación. Dentro de sus hallazgos, los autores comentan que «con este enfoque se logró mantener, a través del período de observación, un clima de aprendizaje cercano a la excelencia» (Domínguez, Sanabria, \& Sierra, 2018, pp. 143144). Estos resultados pueden explicarse por la aplicación de metodologías validadas de bosquejo instruccional cuyos resultados de enseñanza eran esperados, también por el mantenimiento de un entorno virtual atractivo en virtud de las discusiones fluidas entre los participantes, por el buen desempeño del facilitador en el uso de estrategias de enseñanza interactiva, mediante la discusión de casos clínicos, lo cual llevó la teoría a la práctica, y por el uso de herramientas de control de la calidad de la enseñanza. La mayoría de los participantes reflejaron una evaluación positiva a esta estrategia didáctica.

Rivera y García (2018) expresan en su estudio Aula Invertida con tecnologías emergentes en ambientes virtuales en la Universidad Politécnica Salesiana del Ecuador, que el uso del Aula Invertida propicia la generación de aprendizajes pues motiva al estudiante a comprender que él puede tomar control sobre sus propios procesos cognitivos. También, el Aula Invertida potencializa la praxis y es aquí donde el facilitador juega su papel más importante como mediador entre el estudiante, los contenidos, los contextos y los recursos. 
En 2016, Gaspar, Reyes, González y García (2016) desarrollaron el estudio en la Universidad Politécnica Salesiana del Ecuador llamado La gamificación y el Aula Invertida, aplicada en las universidades tecnológicas. Los investigadores resaltan lo siguiente: «El carácter desarrollador de la enseñanza, apoyado en las tecnologías emergentes de la información y las comunicaciones, es la base de los fundamentos de Aula Invertida» (Gaspar et al., 2016, p. 116).

Tomando en consideración que la literatura internacional puntualiza la importancia de implementar estrategias didácticas innovadoras y también de evaluar su utilización desde la perspectiva de los estudiantes, en la presente investigación se llegó a concretar una pregunta central: ¿Cuál es la percepción de los estudiantes con respecto a la implementación del Aula Invertida como estrategia didáctica innovadora en cursos de Mercadeo?

El objetivo general fue el siguiente: analizar la percepción de los estudiantes de los cursos de Seminario de Mercadeo 3 con respecto a la implementación del Aula Invertida como estrategia didáctica innovadora en la Universidad Hispanoamericana. Los objetivos específicos fueron:

a) a) Conocer la percepción de los estudiantes con respecto al rol del facilitador al utilizar el Aula Invertida.

b) b) Describir la percepción de los estudiantes en torno a los conocimientos construidos durante el proceso de aprendizaje mediante Aula Invertida.

c) c) Determinar los beneficios emocionales que surgen a partir de participar en una clase de Aula Invertida.

d) d) Establecer la idoneidad de los instrumentos de evaluación utilizados durante el Aula Invertida.

Rivera y García (2018) explican que los autores de la modalidad Aula Invertida fueron Jonathan Bergmann y Aaron Sams, dos profesores de química en Woodland Park High School en Colorado.
Para comprender el concepto del Aula Invertida, Rodríguez-Carmona y Presol-Herrero (2018) indican que este modelo pedagógico transfiere el trabajo de algunos de los procesos de aprendizaje a contextos fuera del aula, pues se dedica tiempo extra-clase para trabajar algunos conceptos teóricos y aspectos necesarios para los aprendizajes. Según lo anterior, el Aula Invertida optimiza el tiempo en clase, ya que excluye del aula la teoría que, de manera tradicional, se ha aplicado durante el tiempo de clases. Esto permite ampliar la disponibilidad de tiempo tanto para el facilitador como los estudiantes, para el desarrollo de las clases.

Lizcano-Dallos, Barboza-Chacón y -Escobar (2019) comentan lo siguiente sobre las TIC: «...los constructos pedagógicos que sostienen el trabajo colaborativo se orientan al constructivismo; por tanto, las TIC están anidadas a un andamiaje de técnicas, recursos y estrategias educativas» (Lizcano-Dallos et al., 2019, p.18). Las Tecnologías de la Información y la Comunicación son relevantes para la implementación de la modalidad Aula Invertida. Las TIC se conceptualizan como un grupo de herramientas instrumentales que apoyan la labor tanto del estudiante como del facilitador que usan la modalidad Aula Inversa.

Otro aspecto relevante para la aplicación del Aula Invertida es considerar las Tecnologías del Aprendizaje y el Conocimiento, las cuales son definidas por Luque (2016, p.58) de la siguiente forma: «Las TAC podríamos decir que son las TIC pero aplicadas de forma tal que sirven para generar nuevos conocimientos en el alumno (son verdaderas herramientas para el aprendizaje)».

\section{Metodología}

La presente investigación tuvo un enfoque cuantitativo. Según Hernández, Fernández y Baptista (2014, p. 4), el enfoque cuantitativo "utiliza la recolección de datos para probar hipótesis con base en la medición numérica y el análisis estadístico, con el fin establecer pautas de comportamiento y probar teorías". Este estudio tuvo un alcance exploratorio ya que fue la primera vez que se utilizó Aula Invertida en cursos de la Facultad de Ciencias Económicas y se contaba con poca literatura 
costarricenses en torno al tema. Se eligió un diseño no experimental, el cual es entendido por Hernández et al. (2014) como un estudio que se realiza sin manipulación deliberada de las variables. A su vez, el estudio fue transversal pues la información se recolectó en un solo periodo específico.

Los grupos fueron escogidos y asignados al investigador por la Dirección de la Facultad de Administración. Se trató de dos grupos del curso de Seminario de Mercadeo 3, durante el tercer cuatrimestre de 2018.

La Tabla 1 muestra el día en que se impartía el curso, el grupo, el horario y la cantidad de estudiantes matriculados:

Tabla 1: Población

\begin{tabular}{|l|l|l|l|}
\hline Día & Grupo & Horario & Estudiantes \\
\hline Martes & 1 & Nocturno & 15 \\
\hline Viernes & 2 & Diurno & 12 \\
\hline
\end{tabular}

La mayoría de los estudiantes eran solteros con pareja quienes vivían en Heredia y sus alrededores, en algunos casos padres o madres solteras. Eran personas con edades comprendidas entre los 18 y los 30 años. Se contabilizaron catorce hombres y trece mujeres. La muestra utilizada fue de tipo no probabilística. Hernández et al. (2014, p. 176) definen la muestra no probabilística como: "Subgrupo de la población en la que la elección de los elementos no depende de la probabilidad sino de las características de la investigación".

\section{El instrumento de recolección de datos}

El instrumento utilizado fue el cuestionario. Se usaron preguntas cerradas y abiertas, con escalas de diferencial semántico y Likert. Las variables utilizadas en este estudio fueron las siguientes: 1. Desempeño del facilitador 2. Adquisición de los conocimientos 3. Nivel de concentración 4. Nivel de concentración 5. Nivel de retensión de conocimientos. Ahorro de tiempo 7. Calidad de los conocimientos adquiridos 8. Libertad de expresión y 9. Disfrute.
Se realizó un cuestionario basado en Google formulario el cuál se aplicó a los sujetos de estudio. Este constó de veintinueve preguntas, veintiocho cerradas y una abierta. Las escalas utilizadas fueron las siguientes: Diferencial semántico con seis preguntas $(1-4,11$ y 12), escala de Likert con veintidós preguntas (5.1 - 5.5, 6.1 - 6.3, 7.1 7.2, 8.1 - 8.2, 9.1 - 9.7, 10.1 - 10.3) y una pregunta abierta (la número 13).

En cuanto a los cuidados éticos, en la primera lección del curso se procedió a explicar en detalle la metodología de Aula Invertida y a solicitar consentimiento oral de cada uno de los estudiantes. También se explicaron los objetivos del estudio y los deberes y derechos de los participantes: tienen derecho a la libre expresión, a retirarse del estudio y a que sus datos fueran analizados de forma confidencial.

Para las instrucciones se detallaron aspectos esenciales del Aula Invertida: las explicaciones teóricas a lo interno del aula serían mínimas, la teoría se colocaría en la plataforma Moodle, a través de videos. En la clase presencial se realizarían actividades de análisis. Como libros de texto base, se utilizaron Marketing de Kerin (2014), Dirección de Marketing de Lambin (2009) y Marketing de Servicio de Lovelock (2015).

Los temas elegidos fueron los siguientes: creación de valor y de relaciones con los clientes a través del marketing, escanear el entorno de marketing, segmentación, posicionamiento y selección de mercados meta, análisis de la competitividad de la empresa, nuevos enfoques de marketing en la economía de servicios, servicios de posicionamiento en los mercados competitivos, desarrollo de productos de servicios, precios y gestión de ingresos, el diseño y la gestión de los procesos de servicio, creación y gestión de relaciones de lealtad, administración de las quejas y recuperación del servicio, aumento de la calidad y la productividad del servicio.

Se utilizó Moodle como plataforma en línea para presentar los videos y autoevaluaciones. Se realizaron dos preguntas por vídeo. A continuación, se presentan algunos ejemplos de preguntas: 
- ¿Por qué la lealtad de los clientes es importante para la rentabilidad de una empresa?

- ¿Qué efecto tiene en las utilidades, los comentarios "de boca en boca" de clientes desertores?

- ¿Cuáles herramientas se utilizan para comprender la relación entre el cliente y la empresa?

- ¿Qué logra la integración de marketing, operaciones y recursos humanos?

\section{Resultados}

Como primera fase de análisis, se aplicó estadística descriptiva para determinar los valores para las siguientes variables: desempeño del facilitador, calidad de los conocimientos construidos, adquisición de nuevos conocimientos, nivel de concentración, nivel de retención de los conocimientos, ahorro de tiempo, libertad de expresión y disfrute.

El detalle de estos resultados puede observarse en la Tabla 3. En particular, debe destacarse que 95\% de los estudiantes, que participaron en el Aula Invertida, consideraron que la calidad de construcción de conocimientos, la libertad de expresión y el disfrute obtuvieron calificación de excelentes o buenos. De ahí que pueda considerarse que esta estrategia didáctica fue percibida de manera positiva por la mayoría de quienes participaron del estudio, porque resultó un escenario adecuado para la construcción de conocimientos que forman una parte esencial del curso.

En contraposición, los resultados arrojaron que el 66\% consideró que el Aula Invertida les ahorraba tiempo de trabajo extra-clase y $69 \%$ se reportó que ayudaba a memorizar conceptos teóricos propios de la materia. Estos porcentajes, pese a ser inferiores a los de otras variables exploradas, resultan positivos pues el Aula Invertida no pretende promover la retención de los conocimientos de conocimientos ni el ahorro de tiempo. Por el contrario, es una estrategia para que el estudiante participe en su proceso de aprendizaje y dedique más horas fuera del aula para la revisión de los temas propios de la materia.
Tabla 3: Análisis descriptivo de las variables

\begin{tabular}{|c|c|c|c|c|c|c|}
\hline 总 & Escalas & 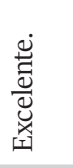 & 宅 & 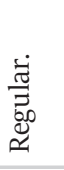 & $\frac{0}{\sum^{\frac{\pi}{z}}}$ & 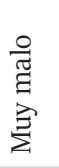 \\
\hline 1 & $\begin{array}{l}\text { Desempeño del } \\
\text { facilitador }\end{array}$ & $69 \%$ & $17 \%$ & $9 \%$ & $\mathrm{O} \%$ & $5 \%$ \\
\hline 2 & $\begin{array}{l}\text { Calidad de los } \\
\text { conocimientos } \\
\text { construidos }\end{array}$ & $39 \%$ & $56 \%$ & $\mathrm{O} \%$ & $\mathrm{O} \%$ & $5 \%$ \\
\hline 3 & $\begin{array}{l}\text { Adquisición de nuevos } \\
\text { conocimientos }\end{array}$ & $65 \%$ & $16 \%$ & $5 \%$ & $5 \%$ & $9 \%$ \\
\hline 4 & Nivel de concentración & $39 \%$ & $35 \%$ & $13 \%$ & $\mathrm{O} \%$ & $13 \%$ \\
\hline 5 & Nivel de retención & $43 \%$ & $26 \%$ & $13 \%$ & $9 \%$ & $9 \%$ \\
\hline 6 & Ahorro de tiempo & $53 \%$ & $13 \%$ & $17 \%$ & $4 \%$ & $13 \%$ \\
\hline 7 & Libertad de expresión & $69 \%$ & $26 \%$ & $5 \%$ & $\mathrm{O} \%$ & $\mathrm{O} \%$ \\
\hline 8 & Disfrute & $52 \%$ & $43 \%$ & $5 \%$ & $\mathrm{O} \%$ & $\mathrm{O} \%$ \\
\hline
\end{tabular}

Fuente: Elaboración propia.

\subsection{Análisis de la tabla 3:}

En esta tabla visualiza que, los factores a la derecha de la tabla, son calificados de manera positiva. Se puede observar algunos casos en los cuales dicha calificación no es tan alta. Esto es notorio en el caso de las siguientes variables: ahorro de tiempo, nivel de retención de los conocimientos y nivel de concentración donde los factores de índole medio (expresado como regular en la tabla) responden a los siguientes porcentajes: $17 \%, 13 \%$ y $13 \%$, respectivamente.

Con el fin de enriquecer la investigación, se crearon gráficos con cruces de variables para profundizar en la medición de las percepciones en torno a los tipos de actividades de evaluación formativa y sumativa que se utilizaron. 


\section{Gráfico 1: Comparativo del nivel de estrés del estudiante versus La calificación al profesor.}

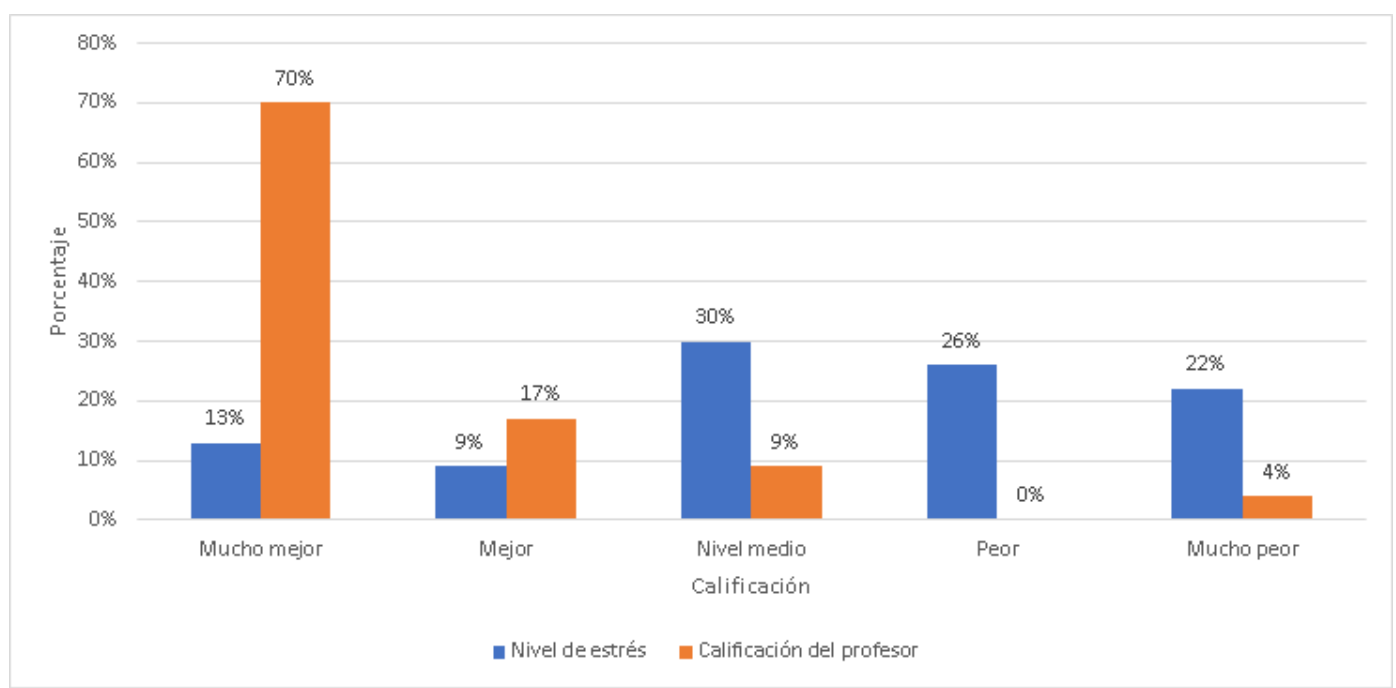

Fuente: Elaboración propia.

Es notable que en las categorías "Mucho Mejor" y "Mejor" con respecto a la relación estrés frente a la calificación del profesor, los estudiantes otorgan una mayor calificación al profesor en el tanto que su nivel de estrés es menor (70\% y $17 \%$ frente al $13 \%$ y $9 \%$ respectivamente). Los sujetos de investigación calificaron peor al profesor en cuanto sus niveles de estrés fueron superiores. Esto se puede observar en las opciones nivel medio, peor y mucho peor (30\%, 26\% y $22 \%$, frente a $9 \%, 0 \%$ y $4 \%$ de manera respectiva)

Gráfico 2: Comparativo sobre la adquisición de conocimientos versus disfrute del aula invertida.

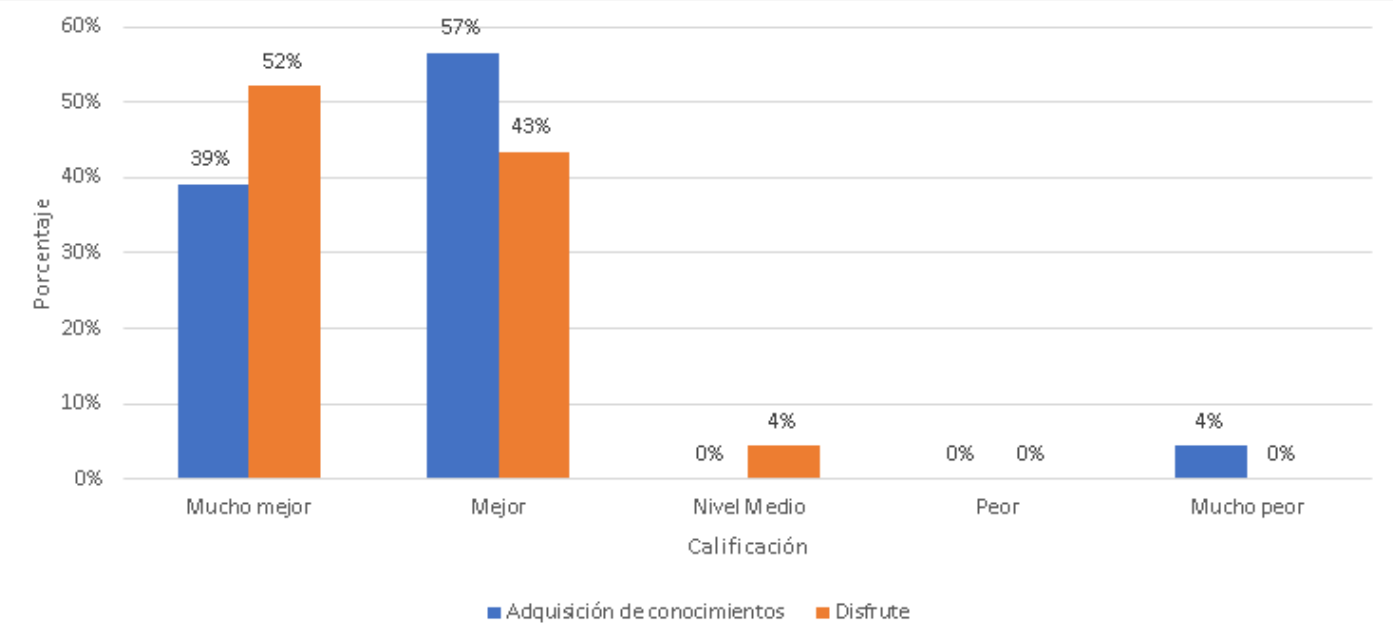

Fuente: Elaboración propia. 
De izquierda a derecha, en las dos opciones de respuesta "mucho mejor y mejor", el nivel de adquisición de conocimientos y el disfrute, se califican, sumando los respectivos porcentajes, en un $96 \%$ frente a un $95 \%$. La calificación del disfrute, en el rubro nivel medio, es más alta que la de la adquisición de conocimientos (un $4 \%$ frente a un $\mathrm{o} \%$ ). Al aplicar sumatoria a las categorías "peor" y "mucho peor" se obtiene un resultado de $4 \%$ para la adquisición de conocimientos frente a un o\% el disfrute del Aula Invertida.

\section{Gráfico 3: Comparativo ahorro de tiempo versus Relevancia de los conocimientos.}

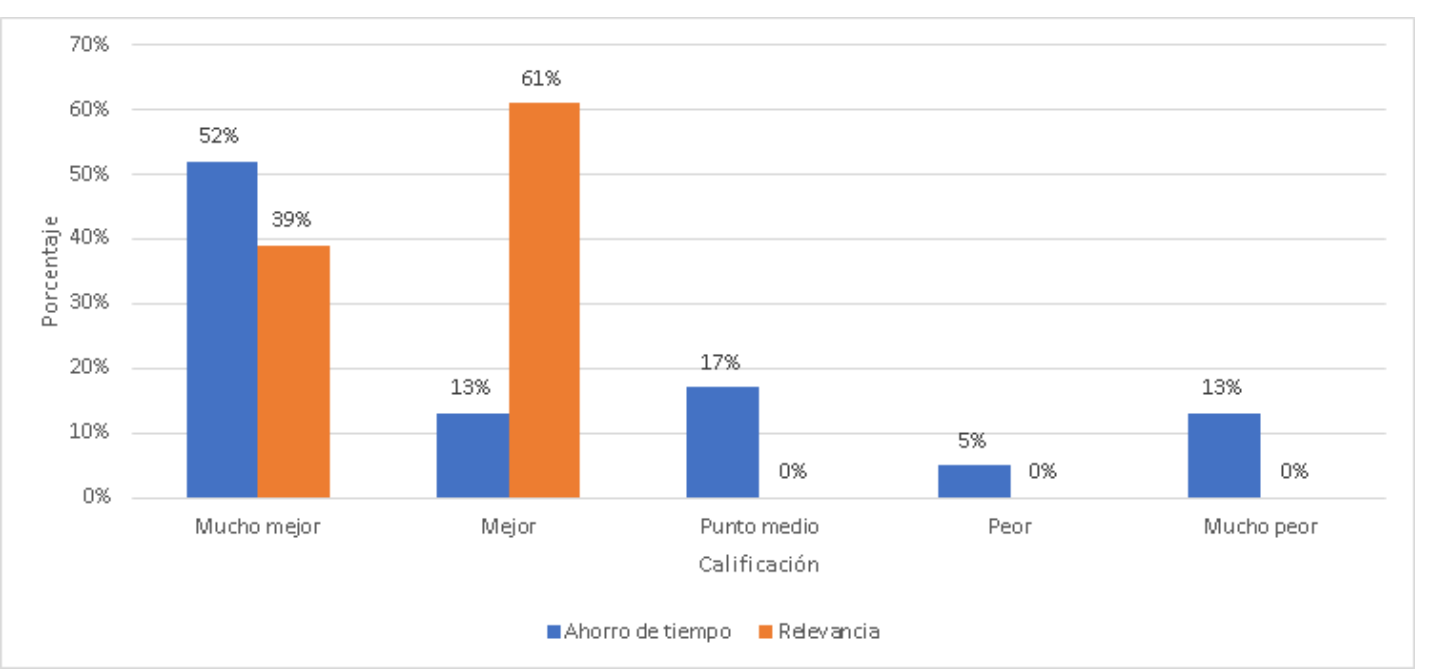

Fuente: Elaboración propia.

La relevancia de conocimientos se polariza hacia las categorías "Mucho Mejor y Mejor". Para los demás, rubros la calificación es igual a o\%. Con respecto a el ahorro de tiempo percibido por parte de los estudiantes, se califica mucho mejor con un $52 \%$, mientras que un $13 \%$ de los estudiantes calificaron como "Mejor".

\section{Gráfico 4: Comparativo de la participación en clases versus la retención de conocimientos.}

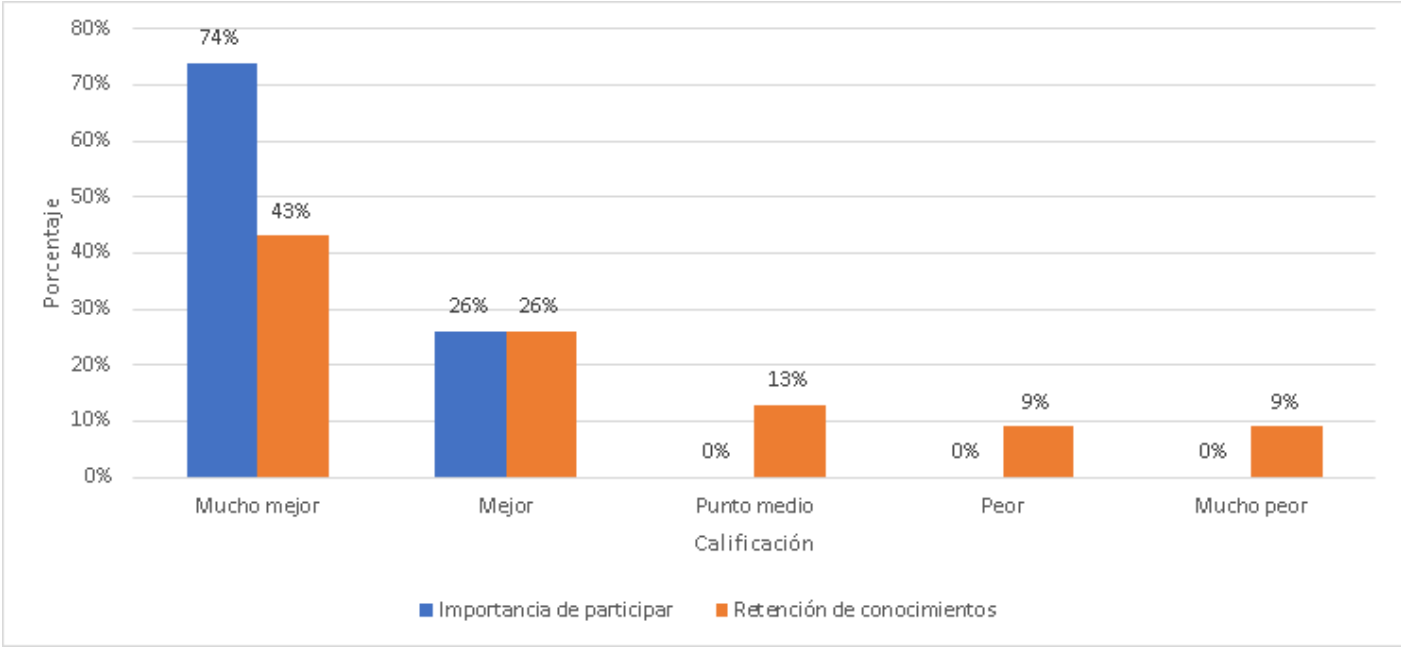

Fuente: Elaboración propia. 
Con respecto a la participación en clases, los estudiantes calificaron con un $74 \%$ de "mucho mejor" y con un $26 \%$ mejor. Por otro lado, la retención de los conocimientos fue calificada en los mismos rubros con un $46 \%$ y un $23 \%$ respectivamente. Se nota un punto medio (regular) con un $13 \%$.

De acuerdo con los datos anteriores, $83 \%$ de las personas entrevistadas evaluaron de mejor forma las actividades asociadas al aprendizaje de conceptos teóricos que otros desarrollados durante el curso. En segundo lugar, de preferencia se encuentran las actividades lúdicas (78\%) y la discusión de casos en el aula (78\%). En tercera posición, las prácticas y los talleres tuvieron la misma calificación: $74 \%$ las consideró muy buenas.

Las pruebas cortas fueron las que obtuvieron la calificación más baja, con un 13\% las calificó como "ni buenas ni malas".

\subsection{Preguntas abiertas:}

En el cuestionario se aplicaron preguntas abiertas para que cada estudiante tuviera la opción de expresar su percepción con respecto a la experiencia de participar en el Aula Invertida.

Algunas de las respuestas más representativas de los participantes a la pregunta abierta, son las siguientes:

- «Este tipo de aprendizaje favorece a los estudiantes, tomando en cuenta los diferentes tipos de aprendizaje, en especial a los quinestésicos. Es posible aprender haciendo, construyendo ideas en clase, apoyados con videos y dinámicas basadas en la vida real.»

- «Me gustó mucho la modalidad, fue mejor; menos aburrido, con más trabajo en casa. En cuanto a los videos, me gustaron mucho, se me facilitó el aprendizaje ya que se explicaba y a la vez se daban ejemplos. En otros cursos nada más se utilizaron diapositivas y esta práctica se torna muy aburrida.»
Dentro de la categoría Disfrute algunos estudiantes expresaron lo siguiente:

- «Un excelente método diferente a los métodos tradicionales de impartir clases. En lo personal, me entretiene más ya que la metodología es más interactiva.»

- «Me gusta mucho el método que se utilizó en clase el cual nos entretiene, nos enseña. Me gustó la parte del análisis de videos.»

Con respecto al acompañamiento del profesor, estos son ejemplos de lo expresado por los estudiantes:

- «Método excelente. Fue de mucha ayuda el conocimiento del profesor. Por favor hacer todos los cursos de esta manera.»

- «Me encantó esta metodología. Es más entretenida y, gracias al profesor, se aprende más con los ejemplos en clase.»

En la categoría Áreas de Oportunidad, estas fueron dos de las percepciones:

- «Es una herramienta útil, pero requiere que las herramientas que se utilicen se expliquen muy bien, sobre todo lo relacionado a la temática.»

- «No siempre tengo el tiempo suficiente para estudiar la materia, pero, ya que está en Moodle, se puede acceder cuando lo necesite.»

Según los expresado, los estudiantes valoraron de forma positiva el curso y, en particular, tuvieron reacciones favorables hacia el uso del Aula Invertida como estrategia didáctica. Esto coincide con los resultados de la gran mayoría de literatura en torno a este tema. A continuación, algunos comentarios representativos de los participantes del estudio:

Sujeto 4: «Me gustó mucho la modalidad ya que en otros cursos se utilizan diapositivas y se torna muy aburrido. El Aula Invertida es mejor, menos aburrido, se da más trabajo 
en equipo. En cuanto a los videos me gustaron mucho, se me facilitó más el aprendizaje ya que se analizaban estos al tiempo de que se enriquecía la discusión con ejemplos.»

Sujeto 8: «Soy de aprendizaje quinestésico, y al verme expuesto a la Aula Invertida, pude lograr un mejor análisis y retentiva de la teoría.»

Sujeto 11: «Me encantó esta metodología. Es más entretenida y se aprende más con los ejemplos que se brindan en la clase. En algunas ocasiones, no me alcanzó el tiempo para estudiar, pero ya que los vídeos y el material ppt están en Moodle, se puede acceder cuando uno desee: ¡Ojalá la implementen en todas las clases! Gracias, profesor.»

Sujeto 13: «Por Favor hacer todos los cursos y pruebas de esta manera, es mucho mejor.»

Sujeto 15: «Es una dinámica de clase muy diferente a lo convencional, es más interesante que las clases normales.»

\section{Discusión}

El presente estudio se centró en las percepciones de los estudiantes de mercadeo 3 al utilizar el Aula Invertida. Se consideró que un factor fundamental dentro de esta modalidad es el rol del facilitador, que en esta ocasión fue considerado de manera positiva. Según Rivera y García (2018), el Aula Invertida potencia la práctica de la teoría y para ello es importante el papel del facilitador. Otro de los aspectos percibidos de forma positiva por los participantes del curso fue transformar las clases magistrales en sesiones prácticas con el uso de Aula Invertida. En particular, cinco participantes expresaron que se habían sentido a gusto pues las clases habían sido dinámicas y hubo posibilidad de aplicar trabajo en grupo y resolución de casos. Esto repercutió en que se diera una sensación de haber aprendido más en este curso que en otros, en palabras de uno de ellos: «Esta metodología me ayudó a entender mejor».

Shih y Tsai (2017) apuntan a que el Aula Invertida ayuda a facilitar la construcción de los aprendizajes en estudiantes. Esto pudo observarse cuando las actividades implementadas lograron un incremento en la concentración del estudiante. Durante las clases, se observó que los estudiantes prestaban atención a los vídeos de casos y a su posterior discusión colectiva. Por otro lado, en los comentarios de los estudiantes se notaba un mayor dominio del tema y el uso de lenguaje más técnico.

En cuanto a la retención de los conocimientos de los contenidos, hay que rescatar lo dicho por Hernández-Silva y Flores (2017), en cuanto a que las clases tradicionales se centran en el contenido y el rol central del profesor, circunscribiéndose en factores memorísticos. Sin embargo, en el cuestionario, la mayoría mencionó que el Aula Invertía ayudaba a memorizar algunos conceptos clave y a entenderlos mejor incluso antes de llegar a la clase. Uno de los participantes expresó: «Esta clase me ayudó a analizar mejor las cosas y tener una mejor retentiva».

El curso de Mercadeo 3 solía caracterizarse por poca participación estudiantil. Esto contrasta con lo sucedido luego de implementar el Aula Invertida: los estudiantes solían hacer más intervenciones en clase y en el cuestionario reportaron sentir mayor libertad de expresión. Esto va en la misma línea de lo planteado por Rivera y García (2018): «el Aula Invertida propicia la generación de contextos de aprendizaje de mayor cercanía con los estudiantes pues son ellos quienes toman el control de su ritmo de aprendizaje».

Otro aspecto que resultó significativo fue el clima lúdico que podía percibirse en el aula. Los estudiantes comentaron que la clase era "relajada" y respondieron en el cuestionario que estaban disfrutando las sesiones de Aula Invertida: «Me gusta mucho el método que se utilizó en clase, este nos entretiene y nos enseña. Me gustó mucho la parte de ver videos». Pese a esto, el disfrute no necesariamente es un facilitador del aprendizaje, pues, en ocasiones, no lleva a la optimización de los procesos cognitivos.

En sintonía con los hallazgos de Domínguez et al. (2018), el presente estudio confirma que el Aula Invertida puede llegar a promover escenarios de aprendizaje favorables. 
Por su parte, Gaspar et al. (2016) comentaron que el sistema educativo busca formar estudiantes competentes y felices siendo esta la razón por la cuál se ha comenzado a prestar más atención a la parte humana y a elementos motivacionales, que se suman a los aprendizajes conceptuales y procedimentales.

Un análisis de la relación entre algunas variables reveló que el nivel de disfrute estuvo mejor calificado que el nivel de concentración, mientras que el de libertad de expresión en clases tuvo el mismo porcentaje que el de disfrute. Comparando esto con los planteamientos de Shih y Tsai (2017), tanto en el estudio de estos autores como en la presente investigación, los estudiantes externaron que el Aula Invertida favorece aprendizajes más efectivos.

Pese a los buenos resultados de la utilización del Aula Invertida con estudiantes de mercadeo, otros estudios advierten que esta estrategia didáctica no siempre da los resultados esperados. Tal es el caso de Schmeisser y Medina (2017, p. 85): «se ha comprobado que la metodología de Aula Invertida no ha producido un mayor impacto en la enseñanza durante los dos meses de investigación». Por otro lado, Scovotti (2016) comenta que su experiencia no fue lo positiva que hubiera esperado pues los estudiantes intervenidos expresaron tanto comentarios negativos como positivos sobre el Aula Invertida. Ella explica que realizó su curso de Principios de Marketing basado en la modalidad Aula Invertida. Al final de su estudio, expresa que los estudiantes se sintieron «desafiados por la carga de trabajo fuera de la clase y se quejaron de que el instructor no les estaba enseñando» (Scovotti, 2016, p. 55).

\section{Conclusiones}

El facilitador cumplió de manera adecuada con su desempeño como guía de los procesos docentes para los estudiantes. Asimismo, logró crear actividades lúdicas en las clases que reforzaron conceptos clave de cada uno de los contenidos del curso. Por otro lado, también logró establecer un ambiente propicio para el aprendizaje.

La calidad de los aprendizajes fue percibida de manera positiva por parte de los estudiantes. En particular, al comparar la construcción de conocimientos en clases magistrales y en clases con modalidad Aula Virtual. En este segundo caso, los participantes del estudio expresaron que era más sencillo asimilar la mayoría de los conocimientos y que, en algunos casos en los que resultaba indispensable memorizar parte del material, fue un proceso menos arduo.

El Aula Invertida también fue considerada una metodología que ayudó a concentrarse mejor frente a temáticas difíciles en clases. Esto según algunos comentarios de estudiantes que indicaron que la presencia de videos en lugar de complejas lecturas ayudaba a concentrarse durante mayor cantidad de tiempo y, así, lograr comprender de una mejor manera el contenido teórico. Los estudiantes analizaban varias veces los vídeos fuera del aula con el fin de llegar a las clases presenciales con una base teórica que les permitiera realizar prácticas y análisis de casos de manera más eficaz.

Un único aspecto fue señalado como negativo en la experiencia del Aula Invertida: el ahorro del tiempo. Varios de los participantes comentaron que esta modalidad tomaba más tiempo del usual para la realización de actividades en sus casas. Tal como se comentó con anterioridad, esta percepción da cuenta de los distintos ritmos de aprendizaje de los estudiantes y es, en el fondo, una de las mayores bondades de la estrategia: aquellas personas que tienen dificultad en algunos contenidos teóricos verán los videos de manera más lenta o en varias ocasiones con el fin de mejorar su comprensión sobre la temática vista. Esto contrasta con lo que sucedería en una clase magistral: todos los estudiantes se verían forzados a ir al mismo ritmo cognitivo.

El facilitador promovió una cultura de análisis de los contenidos, enfatizando en todo momento que había libertad para hacer comentarios y preguntas de la materia. Tener la posibilidad de cuestionar los planteamientos de distintos autores logró reforzar la importancia del pensamiento crítico y de generar un criterio propio en torno a las temáticas. 
Todas las técnicas de evaluación utilizadas durante el curso obtuvieron porcentajes altos de aprobación. Debido al tamaño de la población se advierte que los resultados del presente estudio no se pueden generalizar a poblaciones mayores. Sin embargo, hay una gran riqueza en la interpretación de los datos recolectados, ya que los resultados se pueden extrapolar al resto de la población de la universidad.

Se recomienda realizar futuros estudios con poblaciones de otros cursos de la Facultad de Ciencias Económicas, pues esta área ha tenido poca investigación con respecto a estrategias didácticas innovadoras y su impacto en los niveles de motivación de los estudiantes y en los procesos de construcción de aprendizajes. Se recomienda, además, impulsar a otros profesores de la universidad a realizar estudios sobre el tema.

\section{Referencias}

Albalawi, A., (2018). The effect of using Flipped Classroom in teaching Calculus on students' achievement at the University of Tabuk. International Journal of Research in Education and Science, 4(1), 198-207. doi: 10.2189o/ijres.383137

Domínguez T., Sanabria Q., and Sierra B., (2018). ¿Cómo perciben los estudiantes el clima de aprendizaje en el Aula Invertida en cirugía? Lecciones aprendidas y recomendaciones para su implementación. Revista Chilena de Cirugía, 70(2), 140-146. doi: org/10.4067/s0718-40262018000200140

Gaspar, B., Reyes, M., González, F. \& García, F. (2016) La gamificación y el Aula Invertida, aplicada en las universidades tecnológicas. Revista de Docencia e Investigación Educativa, 2(4), 30-33.

Hernández, R., Fernández, C. \& Baptista, P. (2014). Metodología de la Investigación. Ciudad de México: McGraw-Hill.
Hernández-Silva, C. \& Flores, S. T. (2017). Aula Invertida mediada por el uso de plataformas virtuales: un estudio de caso en la formación de profesores de física. Estudios Pedagógicos (Valdivia), 43(3), 193-204.http://search.ebscohost.com/login.aspx ?direct=true $\& \mathrm{db}=$ eue $\& A N=128210995 \& l a n g=e s$ \&site $=$ eds-live $\&$ scope $=$ site

Jakobsen, K. V. \& Knetemann, M. (2017). Putting structure to Flipped Classroom using team-based learning. International Journal of Teaching and Learning in Higher Education, 29(1), 177-185.

Kerin, H. (2014). Marketing (11 ${ }^{a}$ Ed.). Ciudad de México: McGraw-Hill.

Lambin, J. (2009). Dirección de marketing (2 ${ }^{a}$ Ed.). Ciudad de México: McGraw-Hill.

Lizcano-Dallos, A., Barboza-Chacón, J. \& Villa MizarEscobar, J.D. (2019). Aprendizaje colaborativo con apoyo en TIC: concepto, metodología y recursos. Magis: Revista Internacional de Investigación en Educación, 11(24), 5-24. https:// doi.org/10.11144/Javeriana.m12-24.acat

Lovelock, W. (2015). Marketing de servicio ( $7^{\mathrm{a}}$ Ed.). Ciudad de México: Pearson.

Luque, F. J. (2016). Las TIC en educación: caminando hacia las TAC. Cuadernos de desarrollo aplicados a las TIC, 5(4), 55-62. http://dx.doi. org/10.17993/3ctic.2016.54.55-62/

Melendo, L. \& Presol, A. (2018). La metodología Flipped Classroom en educación superior. Resultados de uso de LYNDA como recurso para las pre-clases. Revista de Comunicación de la SEECI, 46, 77-92. http://dx.doi.org/10.15198/seeci.2018.46.77-92 
Motameni, R. (2018). The combined impact of the Flipped Classroom and Collaborative Learning on students' learning of key marketing concepts. Journal of University Teaching \& Learning Practice, 15(3), 1-21. https://ro.uow.edu.au/jutlp/ vol15/iss $3 / 4$

Reyes-Parra, A., Cañón-Ayala, M. \& Olarte-Dussan, F. (2018). Una propuesta de Aula Invertida en la asignatura de señales y sistemas de la Universidad Nacional de Colombia. Revista Educación en Ingeniería, 13(25), 82-87. https:// doi.org/10.26507/rei.v13n25.877

Rivera, F. M. \& García, A. (2018). Aula Invertida con tecnologías emergentes en ambientes virtuales en la Universidad Politécnica Salesiana del Ecuador. Revista Cubana de Educación Superior, 37(1), 108-123. http://search.ebscohost.com/login. aspx?direct $=$ true $\& \mathrm{db}=\mathrm{asx} \& \mathrm{AN}=128963542 \& \mathrm{l}$ ang $=$ es $\&$ site $=$ eds-live $\&$ scope $=$ site

Rodríguez-Carmona, L. M. \& Presol-Herrero, Á. (2018). Application of LYNDA as a Teaching Resource in the Project of Educational Innovation in the Excellence of Teaching. Revista de Comunicación de La SEECI, 22(46), 77-91. https://doi. org/10.15198/seeci.2018.46.77-92

Schmeisser, C. \& Medina, J. (2017). Efecto del Aula Invertida como Estrategia Didáctica en el Rendimiento Académico. HETS Online Journal, 8, 66-9o. http://search.ebscohost.com/login. aspx?direct $=$ true $\& \mathrm{db}=$ eue $\& A N=126540398 \& \mathrm{l}$ ang $=$ es\&site $=$ eds-live\&scope $=$ site

Scovotti, C. (2016). Experiences with Flipping the Marketing Capstone Course. Marketing Education Review, 26(1), 51-56. http://search.ebscohost. $\mathrm{com} / \operatorname{login}$. aspx?direct=true $\& \mathrm{db}=$ eric $\& A N=\mathrm{EJ} 11$ 31240\&lang $=$ es $\&$ site $=$ eds-live $\&$ scope $=$ site
Wen-Ling, S. \& Chun-Yen, T. (2017). Students' perception of a Flipped Classroom approach to facilitating online Project-based learning in marketing research courses. Australasian Journal of Educational Technology, 3(5). 